Faculty of Science

Faculty Publications

This is a post-review version of the following article:

Electrooxidation of $\mathrm{Pt}(111)$ in acid solution

Jakub Drnec, David A. Harrington \& Olaf M. Magnussen

August 2017

The final published version of this article can be found at:

https://doi.org/10.1016/j.coelec.2017.09.021

Citation for this paper:

Drnec, J., Harrington, D.A. \& Magnussen, O.A. (2017). Electrooxidation of Pt(111) in acid solution. Current Opinion in Electrochemistry, 4(1), 69-75. 
Accepted version of Current Opinion in Electrochem. 4 (2017) 69-75. doi: 10.1016/j.coelec.2017.09.021

(c) 2017. This manuscript version is made available under the CC-BY-NC-ND 4.0 license

http://creativecommons.org/licences/by-nc-nd/4.0/

\title{
Electrooxidation of $\mathrm{Pt}(111)$ in acid solution
}

\author{
Jakub Drnec \\ European Synchrotron Radiation Facility, 71 Avenue des Martyrs, 38000 Grenoble, France \\ David A. Harrington \\ Chemistry Department, University of Victoria, PO Box 1700, Victoria, BC, V8W 2Y2, \\ Canada \\ Olaf M. Magnussen* \\ Institute of Experimental and Applied Physics, Kiel University, Olshausenstr. 40, 24098 \\ Kiel, Germany
}

\begin{abstract}
The electrochemical oxidation of $\mathrm{Pt}$ has regained new interest in recent years, motivated by its importance for Pt electrocatalyst stability. New experimental data obtained by in situ methods, detailed electrochemical studies, and complementary results from ab initio theory and gas phase studies have led to significant advances in the understanding of this process on the atomic scale. Here, an overview of recent work on the electrooxidation of $\mathrm{Pt}(111)$ single crystals in acidic electrolytes, primarily perchloric acid solution, will be given. The complex potential- and time-dependent changes in the adsorbate layer on the electrode surface, the place exchange of oxygen species with Pt surface atoms, the formation of an ultrathin Pt oxide layer, and the structural changes of the electrode surface structure upon oxidation/reduction cycles will be described.
\end{abstract}

Keywords: Platinum, oxidation, atomic processes, $\mathrm{OH}$ adsorption, restructuring, place exchange

\footnotetext{
${ }^{*}$ Corresponding author

Email addresses: jakub@drnec.cz (Jakub Drnec), dharr@uvic.ca (David A. Harrington), magnussen@email.uni-kiel.de (Olaf M. Magnussen)
}

Preprint submitted to Current Opinion in Electrochemistry 


\section{Introduction}

Platinum (Pt) is a key material for electrochemistry, being the most active element for many central electrocatalytic reactions. In particular in electrochemical energy conversion and storage, e.g., in polymer electrolyte membrane

5 fuel cells (PEMFCs), it still is the most important catalyst for applications and remains the benchmark for electrocatalyst materials. The activity and stability of $\mathrm{Pt}$ under electrochemical reactions such as hydrogen evolution, hydrogen oxidation, methanol oxidation, ethanol oxidation, oxygen reduction, and oxygen evolution has been extensively researched in the last decades. 1, 2, 3, 4, 5, 6]

10 In this context, also the oxidation of Pt surface in aqueous environment has received considerable attention, because it is responsible for the diminishing activity towards most surface reactions and for the degradation of $\mathrm{Pt}$ based catalysts. The latter point is especially important for the oxygen reduction reaction in fuel cells and is currently one of the last issues to be solved to make PEMFCs commercially competitive.

Although Pt oxidation and Pt oxide reduction have been studied for a long time, 7, 8, renewed interest in this topic and advances in in situ surface structural characterization and ab initio computational methods have led to new insights and provided a detailed atomistic picture of this important process.

${ }_{20}$ This can serve as a basis for theoretical research, can help to explain many experimental observations, and can guide research into Pt degradation. Here we give a brief overview on the current state of research on Pt oxidation in acid solutions. We will focus on $\mathrm{Pt}(111)$ in the weakly absorbing electrolyte $\mathrm{HClO}_{4}$, but also will provide a brief comparison to the specifically adsorbing case of ${ }_{25} \mathrm{H}_{2} \mathrm{SO}_{4}$. We will also emphasize the locations of the $\mathrm{Pt}$ atoms in the various structures, which have been relatively neglected in earlier literature.

The potential-dependent oxidation processes are summarized in Fig. 1, together with the corresponding cyclic voltammograms (CVs). The detailed behavior in the different potential regions (all given with respect to the reversible hydrogen electrode) will be discussed in the following sections. 


\section{H UPD and butterfly regions}

The surface at $0.05 \mathrm{~V}$ has $2 / 3 \mathrm{ML}$ of underpotentially adsorbed $\mathrm{H}$ atoms and a lattice expansion of about 1-2\% between the first and second Pt layers [9, 10, 11, 12, similar to the expansion on a clean surface in vacuum. 13] Desorption

35 of $\mathrm{H}$ over the range $0.05-0.4 \mathrm{~V}$ reduces the interlayer expansion to about $1 \%$ or less. 12, 14, 9, 10, This range is followed by the double region $0.40-0.60 \mathrm{~V}$, over which the interlayer spacing is constant. No adsorption occurs in this region. [15]

The "butterfly" region, $0.60-0.95 \mathrm{~V}$ in $\mathrm{HClO}_{4}$, consists of two parts, the sharp peaks at $0.8 \mathrm{~V}$ and the broader region leading up to them. These are 40 due to adsorption and desorption of adsorbed $\mathrm{OH}$. The assignment to $\mathrm{OH}$ is from the lack of shift vs RHE as the $\mathrm{pH}$ is changed, consistent with oneelectron oxidation of water to $\mathrm{H}^{+}$and adsorbed $\mathrm{OH}$, and a thermodynamic analysis that shows transfer of one electron per adsorbed OH.[16] However, the perchlorate concentration dependence implies involvement of perchlorate, 45 and it has recently been suggested that perchlorate anions specifically adsorb in competition with $\mathrm{OH},[17$ or that perchlorate in the double layer strongly interacts with adsorbed $\mathrm{OH} .[18$, though chloride contamination can confound the interpretation in perchloric acid. [19, 20]

In the case of sulfuric acid electrolyte, the adlayer structure in the butterfly region is well established by concentration dependence studies, 21, 22. radiochemistry, 23] STM[24, 25] and infrared spectroscopy [26] to be due to specific adsorption of $0.2 \mathrm{ML}$ of sulfate or bisulfate, with uncertainty about the exact species. The thermodynamic analysis yields an approximate charge number of 2, suggesting sulfate, but this number has to be carefully interpreted 55 in the light of possible coadsorption. 27. Infrared spectroscopy suggested that bisulfate predominates for $\mathrm{pH}<3.3,[26$, while later revision by careful thermodynamic analysis showed that sulfate is preferentially adsorbed in the whole butterfly region for $0.8<\mathrm{pH}<3.8$. [22]

The sharp peaks are associated with a fast phase transition, where the cov${ }_{60}$ erage of a disordered phase increases in the broad region and transitions to an 
ordered adlayer structure in the sharp peak. [28, 29] In the case of $\mathrm{H}_{2} \mathrm{SO}_{4}$, the phase transition is from disordered bidentate (bi)sulfate to an ordered $(\sqrt{3} \times \sqrt{7})$ overlayer of tridentate (bi)sulfate with coadsorbed water, and the phase transition has been directly observed by STM[30, SFG [31] and SXRD 12.

There is less direct evidence for the phase transition for $\mathrm{HClO}_{4}$. Recent in situ SXRD studies show a continuous increase in the coverage of oxygen species $\left(\mathrm{OH}_{\mathrm{ads}}, \mathrm{H}_{2} \mathrm{O}, \mathrm{H}_{3} \mathrm{O}^{+}\right)$and a concomittant relaxation of the spacing of the topmost $\mathrm{Pt}$ layer in the entire double layer regime.12. In accordance with results for $\mathrm{Pt}(111)$ oxidation in the gas phase and DFT calculations, Kondo et al. reported a $\mathrm{p}(2 \times 2)$ phase in their in situ SXRD studies and assigned it to a mixed layer of $\mathrm{OH}_{\text {ads }}$ and $\mathrm{H}_{2} \mathrm{O}$. Furthermore, they observed in potentialdependent measurements of the non-specular crystal truncation rod a distinct two-step process. This behavior was attributed to random adsorption in the broad region, followed by the formation of a transient $\mathrm{p}(2 \times 2)$ adsorbate phase and a subsequent phase transition to a $\mathrm{p}(1 \times 1)$ adlayer at the sharp peak. SXRD studies by Liu et al. found in addition the formation of a buckled top Pt layer in the butterfly region, 32 in a good agreement with DFT predictions. 33] Both authors modeled their data with the oxygen atoms in fcc or hcp hollow sites, as suggested by DFT 34] and gas-phase studies [35, 13, though with different coverages.

Although the theory for the order-disorder phase transition explains both the broad and sharp component components of the peak with one type of adsorbate, it has also been suggested that the two components could result from $\mathrm{OH}$ interacting with two types of water species.19 More detailed kinetic studies suggest there may be additional complexity. 36, 37.

At the end of the butterfly peak at $0.90 \mathrm{~V}$, electrochemical measurements suggest an OH coverage of 0.5 ML. [36, 37, 16] In contrast, DFT calculations suggested that $\mathrm{OH}$ coverages cannot exceed 0.33 ML until much higher potentials. [38. This has led to suggestions that some of the butterfly coverage may be $\mathrm{O}$ rather 90 than $\mathrm{OH}$, which is supported by photoelectron spectroscopy (XPS). 39. However, Farkas et al. have pointed out the neglected role of Pt surface buckling 
for coverages above 0.4 ML that may reduce repulsion between oxygen species and enable higher coverages at lower potentials than otherwise. 40] Alltogether, the exact composition of the layer in this regime is still unknown. As described above, all current studies propose an adlayer containing different oxygen species, but with different surface stoichiometries.

\section{Pt oxide peak}

The anodic peak at $\approx 1.05 \mathrm{~V}$ is traditionally assigned to the further oxidation of $\mathrm{OH}_{\text {ads }}$ to $\mathrm{O}_{\mathrm{ads}}$. 36] However, first in situ SXRD measurements on the

100

structure of the oxidized Pt surface by You et al. showed that place exchange $(\mathrm{PE})$, in which a $\mathrm{Pt}$ surface atom exchanges with an oxygen species, occurs in the potential region of this peak. 41] This process is structurally reversible, in the sense that cycling returns the Pt atoms to their original state, and thus the voltammogram is stable for many cycles at typical sweep rates. Later, the same group presented cycling experiments, 42] which showed hysteresis in the SXRD intensity that paralleled the asymmetric voltammetry peaks. Unfortunately, these data have been largely ignored in the literature. Most discussions of this peak considered only adlayer structures and neglected the role of Pt surface restructuring, which was assumed only to be important at higher potentials.

With advances in synchrotron-based techniques parallel voltammetric and SXRD studies are nowadays possible, enabling direct correlation between the structural and the electrochemical data (Fig. 2). 14, 32] These clearly confirm the earlier SXRD results, demonstrating unambiguously that the onset of X-ray intensity changes due to PE occurs precisely at the Pt oxide peak. 14] Furthermore, they revealed a common log time dependence of the PE coverage determined from electrochemical measurements and from the intensity changes. The correlation of PE with an electrochemical current is not obvious, since in principle, the translocation of the Pt atom need not necessarily be concerted with electron transfer or motion of an oxygen-containing species. It suggests that the $\mathrm{PE}$ may occur in the composite process $\mathrm{OH}($ ads $)+\mathrm{Pt} \rightarrow \mathrm{PtO}($ exchanged $)+$ 
$\mathrm{H}^{+}+\mathrm{e}^{-}$, probably with motion of the $\mathrm{Pt}$ as the rate-determining step. Much of the literature referring to the simpler $\mathrm{OH}($ ads $) \rightarrow \mathrm{O}($ ads $)+\mathrm{H}^{+}+\mathrm{e}^{-}$may be reinterpreted as the net reaction above, though there may be conditions under which the $\mathrm{Pt}, \mathrm{O}$, and electron motions are not as tightly coupled.

Interestingly, the anodic Pt oxide peak has no cathodic counterpart and the current corresponding to the reduction is distributed over a broad potential range between $1.00 \mathrm{~V}$ and $0.80 \mathrm{~V}$. This is corroborated by the in situ SXRD measurements, which show a slow recovery of the intensity over this potential range (Fig. 2b). Based on electrochemical measurements, it has been concluded that multiple Pt oxide species, $\mathrm{OH}_{\mathrm{ads}}, \mathrm{O}_{\mathrm{ads}}$, and subsurface oxygen $\left(\mathrm{O}_{\text {sub }}\right)$, inter-convert in this potential region and that the relative coverages strongly depend on the total surface coverages rather than the potential. [36, 37] The hysteresis between oxidation and reduction is apparently an effect of the stability of the oxide, which depends on the overall coverages of the oxygenated species. 135 Specifically, it was suggested higher coverage results in a greater stability and subsequently in a less positive reduction potential. 36, 37.

Electrochemical studies also revealed an influence of both $\mathrm{pH}$ and $\mathrm{ClO}_{4}{ }^{-}$concentration on the position of the anodic peak. This has been partially explained by the competition of the $\mathrm{ClO}_{4}$ adsorption and $\mathrm{H}_{2} \mathrm{O}$ oxidation pathways. [17] $\mathrm{ClO}_{4}$ presence in the vicinity of the surface and in the potential window of the anodic oxide peak has been confirmed. [18]

For low coverages of place exchanged $\mathrm{Pt}$ atoms $\left(\mathrm{Pt}_{\text {exch }}\right)$ the $\mathrm{Pt}(111)$ surface structure fully recovers after oxide reduction. In this case, the $\mathrm{Pt}_{\text {exch }}$ do not interact laterally and apparently can directly move back into their original lattice site upon reduction. According to detailed SXRD measurements (Fig. 2a), 14] the $\mathrm{Pt}_{\text {exch }}$ are exactly located above the vacancy sites, created in the $\mathrm{Pt}$ lattice during PE. This position most likely is stabilized by oxygen species in the vacancy as well as on the surrounding Pt surface and may explain the high reversibility of the place exchange process. As a consequence of the slow PE kinetics, fast cycling up to $1.20 \mathrm{~V}$ still does not result in a $\mathrm{Pt}_{\text {exch }}$ coverage above 
the critical value and the CV thus is reproducible during subsequent cycles. Also the apparent non-reversibility of the anodic peak can be attributed to this slow PE kinetics.

From the above results it can be concluded that the "more stable oxide" responsible for the anodic peak asymmetry and hysteresis [36] and the platinumperoxo-like and platinum-superoxo-like 2D surface oxides reported in Raman spectroscopy measurements, 18 refer to a Pt surface partly covered by (isolated) $\mathrm{Pt}_{\text {exch. }}$. Although this geometry was not considered in the study by Huang et al., such spectroscopic data should shed light on the local Pt-O bonding of the place exchanged atoms.

Further insight into the PE process comes from recently DFT studies of Pt(111) oxidation. 43, 44] According to these calculations the Pt extraction, i.e., the first step of the $\mathrm{PE}$, depends on i) the local $\mathrm{O}_{\text {ads }}$ coverage where 3 adjacent fcc adsorption sites need to be occupied and ii) the presence of surface $\mathrm{H}_{2} \mathrm{O}$, which stabilizes the $\mathrm{Pt}_{\text {exch }}$. Therefore, $\mathrm{Pt}$ extraction is favored for (local) $\mathrm{O}_{\text {ads }}$ coverages $>0.5 \mathrm{ML}$ while the subsequent $\mathrm{O}$ substitution in the resulting hole is favored at coverages $>0.75 \mathrm{ML}$.

\section{D oxide film and surface restructuring}

Increasing the potential for extended times above $1.15 \mathrm{~V}$ leads to irreversible changes in the CVs for all $\mathrm{Pt}$ surfaces. In both $\mathrm{HClO}_{4}$ and $\mathrm{H}_{2} \mathrm{SO}_{4}$ this results in the growth of sharp peaks in the $\mathrm{H}_{\mathrm{UPD}}$ region assigned to $\mathrm{H}$ adsorption on $\{100\}-$ and $\{110\}$-like defects. 45] The specific adsorption of anions has two seemingly contradictory effects: high sulfate concentrations decrease the speed of the surface reordering, while low concentrations increase this speed. This has been assigned to the role of water dissociation during oxidation. 45]

Recent in situ SXRD studies provided detailed data on the potential-dependent structure of the (steady-state) oxide film on $\mathrm{Pt}(111)$ in $\mathrm{HClO}_{4}$, formed by keeping the potential for several minutes in this range. 46. Between 1.17 and $1.62 \mathrm{~V}$ a continuous transition from a surface covered by a high $\mathrm{Pt}_{\text {exch }}$ coverage $(\leq 0.2$ 
ML) to a rather uniform, $\approx 5$ Åthick oxide layer was observed. Simultaneously, the Pt surface atoms in the two layers of the Pt oxide became gradually more disordered, indicating a largely amorphous structure at the upper potential limit. These observations are in qualitative agreement with the Raman spectroscopy data at these potentials, which report a $3 \mathrm{D} \alpha-\mathrm{PtO}_{2} \cdot 18$

The interaction between neighboring $\mathrm{Pt}_{\text {exch }}$ and the decreasing lattice order are most likely responsible for the irreversibility in the oxidation/reduction process in this range. As shown previously, the latter leads to the formation of a roughened surface, covered by nanoscale Pt islands. 47. The structural reordering induced by oxidation/reduction cycles, was recently studied in detail by in-situ grazing incidence small angle scattering (GISAXS). 48, This technique allowed quantitative characterization of the nanoscale surface morphology as a function of upper potential limit and the number of cycles (Fig. 3a). The results indicate a continuous increase in the height of the nanoscale islands and a more and more homogeneous island distribution with increasing number of cycles, whereas the characteristic distances between the islands predominantly dependent on the potential limit. This growth dynamics resembles the one observed in Pt deposition and surface erosion experiments under UHV conditions. It can be rationalized by the nucleation and growth of $\mathrm{Pt}$ adatoms and vacancies, formed on the surface after oxide reduction.

Finally, the oxidation behavior near steps and defect sites may be distinctly differ from that of atomically smooth low-index Pt surfaces. In operando GISAXS studies, obtained during the cycling of $\mathrm{Pt}(111)$ electrodes already roughened by previous potential cycles, indicated an onset of Pt restructuring at $0.80 \mathrm{~V}$, which was attributed to step edge oxidation, followed by more pronounced restructuring above $1.05 \mathrm{~V}$, i.e., the potential of $\mathrm{PE}$ on $\mathrm{Pt}(111)$ terraces (Fig. 3b). [46] 


\section{Conclusion}

In recent years, Pt electrochemical oxidation has received renewed interest,

210

catalysts. Detailed studies by state-of-the-art in situ techniques and ab initio theory provided an atomistic picture of the surface during oxidation and new insight into the elementary mechanisms of oxidation and oxide reduction. A complex sequence of potential-dependent changes in the chemical composition

of the adsorbate layer and the structural arrangement of the Pt surface atoms has emerged from this work. Specifically, gradual changes in the Pt-Pt bonding at the surface have been revealed, starting with the relaxation and buckling of the surface layer and progressing with the slow formation of place-exchanged atoms up to a saturation coverage, which approaches $0.5 \mathrm{ML}$ at potentials of ${ }_{220} \approx 1.6 \mathrm{~V}$, corresponding to a well-defined $\approx 5$ Åthick Pt oxide. Apart from its basic importance for Pt electrochemistry, these results are also instrumental in order to improve the Pt catalyst stability in PEMFCs.

\section{Acknowledgements}

We thank M. Ruge for help in preparing the figures. OMM gratefully 225 acknowledges financial support by the Deutsche Forschungsgemeinschaft via MA1618/13 and MA1618/20. DAH thanks NSERC for financial support.

\section{References}

[1] A. M. Gómez-Marín, R. Rizo, J. M. Feliu, Oxygen reduction reaction at Pt single crystals: a critical overview, Catal. Sci. Technol. 4 (6) (2014) 1685. doi:10.1039/c3cy01049j.

[2] M. T. M. Koper, Structure sensitivity and nanoscale effects in electrocatalysis, Nanoscale 3 (2011) 2054-2073. doi:10.1039/CONR00857E 
[3] A. R. Zeradjanin, J.-P. Grote, G. Polymeros, K. J. J. Mayrhofer, A critical review on hydrogen evolution electrocatalysis: Re-exploring the volcanorelationship, Electroanalysis 28 (10) (2016) 2256-2269. doi:10.1002/ elan.201600270.

[4] M. Akhairi, S. Kamarudin, Catalysts in direct ethanol fuel cell (defc): An overview, International Journal of Hydrogen Energy 41 (7) (2016) 4214 4228. doi:http://dx.doi.org/10.1016/j.ijhydene.2015.12.145.

[5] A. Chen, P. Holt-Hindle, Platinum-based nanostructured materials: Synthesis, properties, and applications, Chemical Reviews 110 (6) (2010) 37673804. doi:10.1021/cr9003902.

[6] I. Katsounaros, S. Cherevko, A. R. Zeradjanin, K. J. J. Mayrhofer, Oxygen electrochemistry as a cornerstone for sustainable energy conversion, Angewandte Chemie International Edition 53 (1) (2014) 102-121.

[7] B. Conway, Electrochemical oxide film formation at noble metals as a surface-chemical process, Progress in Surface Science 49 (4) (1995) 331 - 452. doi:http://dx.doi.org/10.1016/0079-6816(95)00040-6.

[8] V. Climent, J. M. Feliu, Thirty years of platinum single crystal electrochemistry, Journal of Solid State Electrochemistry 15 (7) (2011) 1297. doi:10.1007/s10008-011-1372-1.

[9] I. Tidswell, N. Markovic, P. Ross, Potential dependent surface structure of the $\mathrm{Pt}(111)$ electrolyte interface, Journal of Electroanalytical

घ Chemistry 376 (1) (1994) 119 - 126. doi:http://dx.doi.org/10.1016/ 0022-0728(94) 03553-9.

[10] N. Markovic, P. Ross, Electrocatalysts by design: from the tailored surface to a commercial catalyst, Electrochimica Acta 45 (25) (2000) $4101-4115$. doi:http://dx.doi.org/10.1016/S0013-4686(00)00526-0.

[11] Y. Liu, A. Barbour, V. Komanicky, H. You, X-ray crystal truncation rod studies of surface oxidation and reduction on Pt(111), The Journal of Phys- 
ical Chemistry C 120 (29) (2016) 16174-16178. doi:10.1021/acs.jpcc. 6b00492

[12] T. Kondo, T. Masuda, N. Aoki, K. Uosaki, Potential-Dependent Structures and Potential-Induced Structure Changes at Pt(111) Single-Crystal Electrode/Sulfuric and Perchloric Acid Interfaces in the Potential Region between Hydrogen Underpotential Deposition and Surface Oxide Formation by In Situ Surface Xray Scattering, J. Phys. Chem. C (111) (2016) null. doi:10.1021/acs.jpcc.5b12766.

[13] N. Materer, U. Starke, A. Barbieri, R. Döll, K. Heinz, M. Van Hove, G. Somorjai, Reliability of detailed LEED structural analyses: Pt(111)

口 and Pt(111)-p(2x2)-O, Surface Science 325 (3) (1995) 207 - 222. doi: http://dx.doi.org/10.1016/0039-6028(94)00703-9.

[14] J. Drnec, M. Ruge, F. Reikowski, B. Rahn, F. Carlà, R. Felici, J. Stettner, O. M. Magnussen, D. A. Harrington, Initial stages of $\operatorname{Pt}(111)$ electrooxidation : dynamic and structural studies by surface X-ray diffraction, Elec-

口 trochim. Acta 224 (2017) 220-227. doi:10.1016/j.electacta.2016.12. 028.

[15] M. T. Koper, Blank voltammetry of hexagonal surfaces of pt-group metal electrodes: Comparison to density functional theory calculations and ultra-high vacuum experiments on water dissociation, Electrochimica Acta 56 (28) (2011) 10645 - 10651, selected Papers from the 61st ISE Meet-

₫ ing, Nice, France, 2010. doi:http://dx.doi.org/10.1016/j.electacta. 2011.02 .001

[16] J. Mostany, E. Herrero, J. M. Feliu, J. Lipkowski, Determination of the Gibbs excess of $\mathrm{H}$ and $\mathrm{OH}$ adsorbed at a $\mathrm{Pt}(111)$ electrode surface using a thermodynamic method, Journal of Electroanalytical Chemistry 558 (2003) 19 - 24. doi:http://dx.doi.org/10.1016/S0022-0728(03)00372-3.

[17] G. A. Attard, A. Brew, K. Hunter, J. Sharman, E. Wright, Specific ad- 
sorption of perchlorate anions on $\mathrm{Pt}\{\mathrm{hkl}\}$ single crystal electrodes, Phys. Chem. Chem. Phys. 16 (27) (2014) 13689. doi:10.1039/c4cp00564c.

[18] Y.-F. Huang, P. Kooyman, M. T. M. Koper, Intermediate stages of electrochemical oxidation of single-crystalline platinum revealed by in situ Raman spectroscopy, Nat. Commun. 7 (2016) 12440. doi:10.1038/ncomms12440

[19] A. Berna, V. Climent, J. M. Feliu, New understanding of the nature of OH adsorption on $\mathrm{Pt}(111)$ electrodes, Electrochemistry Communications 9 (12) (2007) 2789 - 2794. doi:http://dx.doi.org/10.1016/j.elecom.2007. 09.018

[20] D. V. Tripkovic, D. Strmcnik, D. van der Vliet, V. Stamenkovic, N. M. Markovic, The role of anions in surface electrochemistry, Faraday Discuss. 140 (2009) 25-40. doi:10.1039/B803714K.

[21] K. Jaaf-Golze, D. Kolb, D. Scherson, On the voltammetry of curves of Pt (111) in aqueous solutions, Journal of Electroanalytical Chemistry and 口Interfacial Electrochemistry 200 (1) (1986) 353 - 362. doi:http://dx. doi.org/10.1016/0022-0728(86)90067-7.

[22] N. Garcia-Araez, V. Climent, P. Rodriguez, J. M. Feliu, Elucidation of the chemical nature of adsorbed species for $\mathrm{Pt}(111)$ in $\mathrm{H}_{2} \mathrm{SO}_{4}$ solutions

n by thermodynamic analysis, Langmuir 26 (14) (2010) 12408-12417. doi: $10.1021 / \mathrm{la} 101112 \mathrm{~b}$

[23] A. Kolics, A. Wieckowski, Adsorption of Bisulfate and Sulfate Anions on a Pt(111) Electrode, The Journal of Physical Chemistry B 105 (13) (2001) 2588-2595. doi:10.1021/jp003536f

[24] A. Funtikov, U. Linke, U. Stimming, R. Vogel, An in-situ STM study of anion adsorption on $\mathrm{Pt}(111)$ from sulfuric acid solutions, Surface Science 324 (1) (1995) L343 - L348. doi:http://dx.doi.org/10.1016/ 0039-6028(94)00774-8. 
[25] A. Funtikov, U. Stimming, R. Vogel, Anion adsorption from sulfuric acid solutions on Pt(111) single crystal electrodes, Journal of Electroanalytical Chemistry 428 (1) (1997) 147 - 153. doi:http://dx.doi.org/10.1016/ S0022-0728(96)05051-6.

[29] M. T. Koper, J. J. Lukkien, Modeling the butterfly: influence of lateral interactions and adsorption geometry on the voltammetry at (111) and

[26] A. Lachenwitzer, N. Li, J. Lipkowski, Determination of the acid dissociation constant for bisulfate adsorbed at the $\mathrm{Pt}(111)$ electrode by subtractively normalized interfacial Fourier transform infrared spectroscopy, Journal of Electroanalytical Chemistry 532 (1) (2002) 85 - 98. doi:http://dx.doi. org/10.1016/S0022-0728(02)00759-3.

[27] E. Herrero, J. Mostany, J. M. Feliu, J. Lipkowski, Thermodynamic studies of anion adsorption at the $\mathrm{Pt}(111)$ electrode surface in sulfuric acid solutions, Journal of Electroanalytical Chemistry 534 (1) (2002) 79 - 89. doi:http://dx.doi.org/10.1016/S0022-0728(02)01101-4.

[28] M. T. Koper, J. J. Lukkien, Modeling the butterfly: the voltammetry of $(\sqrt{3} \times \sqrt{3}) \mathrm{R} 30^{\circ}$ and $\mathrm{p}(2 \times 2)$ overlayers on (111) electrodes, Journal of Electroanalytical Chemistry 485 (2) (2000) 161 - 165. doi:http://dx. doi.org/10.1016/0079-6816(95)00040-6 (100) electrodes, Surface Science 498 (1) (2002) 105 - 115. doi:http: //dx.doi.org/10.1016/S0039-6028(01)01681-8

[30] B. Braunschweig, W. Daum, Superstructures and orderdisorder transition of sulfate adlayers on $\mathrm{Pt}(111)$ in sulfuric acid solution, Langmuir 25 (18) (2009) 11112-11120. doi:10.1021/la901399j.

[31] B. Braunschweig, P. Mukherjee, D. D. Dlott, A. Wieckowski, Real-time investigations of $\mathrm{Pt}(111)$ surface transformations in sulfuric acid solutions, Journal of the American Chemical Society 132 (40) (2010) 14036-14038. doi:10.1021/ja106618z. 
[32] Y. Liu, A. Barbour, V. Komanicky, H. You, X-ray Crystal Truncation Rod Studies of Surface Oxidation and Reduction on Pt(111), J. Phys. Chem. C 0 (111) (2016) acs.jpcc.6b00492. doi:10.1021/acs.jpcc.6b00492.

[33] E. F. Holby, J. Greeley, D. Morgan, Thermodynamics and hysteresis of oxide formation and removal on platinum (111) surfaces, J. Phys. Chem. C 116 (18) (2012) 9942-9946. doi:10.1021/jp210805z.

[34] A. Bogicevic, J. Strömquist, B. I. Lundqvist, First-principles diffusionbarrier calculation for atomic oxygen on Pt(111), Phys. Rev. B 57 (1998) R4289-R4292. doi:10.1103/PhysRevB.57.R4289.

[35] U. Starke, N. Materer, A. Barbieri, R. Döll, K. Heinz, M. Van Hove, G. Somorjai, A low-energy electron diffraction study of oxygen, water and ice adsorption on Pt(111), Surface Science 287 (1993) 432 - 437. doi:http://dx.doi.org/10.1016/0039-6028(93)90818-5.

[36] A. M. Gómez-Marín, J. Clavilier, J. M. Feliu, Sequential Pt(111) oxide formation in perchloric acid: An electrochemical study of surface species inter-conversion, J. Electroanal. Chem. 688 (2013) 360-370. doi:10.1016/ j.jelechem.2012.07.016.

[37] A. M. Gómez-Marín, J. M. Feliu, Oxide growth dynamics at Pt(111) in absence of specific adsorption: A mechanistic study, Electrochim. Acta 104 (2013) 367-377. doi:10.1016/j.electacta.2012.10.075.

[38] A. S. Bondarenko, I. E. L. Stephens, H. A. Hansen, F. J. Perez-Alonso, V. Tripkovic, T. P. Johansson, J. Rossmeisl, J. K. Norskov, I. Chorkendorff, The $\mathrm{Pt}(111) /$ electrolyte interface under oxygen reduction reaction conditions: An electrochemical impedance spectroscopy study, Langmuir 27 (5) (2011) 2058-2066. doi:10.1021/la1042475.

[39] M. Wakisaka, H. Suzuki, S. Mitsui, H. Uchida, M. Watanabe, Identification 370 and quantification of oxygen species adsorbed on $\mathrm{Pt}(111)$ single-crystal 
and polycrystalline pt electrodes by photoelectron spectroscopy, Langmuir 25 (4) (2009) 1897-1900. doi:10.1021/la803050r.

[40] A. Farkas, D. Fantauzzi, J. E. Mueller, T. Zhu, C. Papp, H.-P. Steinrück, T. Jacob, On the platinum-oxide formation under gas-phase and electrochemical conditions, Journal of Electron Spectroscopy and Related Phenomena (2017)-doi:https://doi.org/10.1016/j.elspec.2017.06. 005 .

[41] H. You, D. J. Zurawski, Z. Nagy, R. M. Yonco, In-situ x-ray reflectivity study of incipient oxidation of $\mathrm{Pt}(111)$ surface in electrolyte solutions, The 380 \ Journal of Chemical Physics 100 (6) (1994) 4699-4702. doi:10.1063/1. 466254

[42] Z. Nagy, H. You, Applications of surface x-ray scattering to electrochemistry problems, Electrochimica Acta 47 (19) (2002) 3037 - 3055. doi:http://dx.doi.org/10.1016/S0013-4686(02)00223-2.

[43] M. J. Eslamibidgoli, M. H. Eikerling, Atomistic Mechanism of Pt Ex$\square$ traction at Oxidized Surfaces: Insights from DFT, Electrocatalysisdoi: $10.1007 / \mathrm{s} 12678-016-0313-2$

[44] M. J. Eslamibidgoli, M. H. Eikerling, Electrochemical formation of reactive oxygen species at Pt (111)a density functional theory study, ACS Catalysis 5 (10) (2015) 6090-6098. doi:10.1021/acscatal.5b01154.

[45] A. M. Gómez-Marín, J. M. Feliu, Pt(111) surface disorder kinetics in perchloric acid solutions and the influence of specific anion adsorption, Electrochim. Acta 82 (2012) 558-569. doi:10.1016/j.electacta.2012.04. 066.

395 [46] M. Ruge, J. Drnec, B. Rahn, F. Reikowski, D. Harrington, F. Carl, R. Felici, J. Stettner, O. Magnussen, Electrochemical oxidation of smooth and nanoscale rough Pt(111): An in situ surface x-ray scattering study, J. Electrochem. Soc 164 (2017) H608-H614. doi:10.1149/2.0741709jes. 
[47] K. Itaya, S. Sugawara, K. Sashikata, N. Furuya, In situ scanning tunneling microscopy of platinum (111) surface with the observation of monoatomic steps, J. Vac. Sci. Technol. A 8 (1990) 515.

[48] M. Ruge, J. Drnec, B. Rahn, F. Reikowski, D. A. Harrington, F. Carlà, R. Felici, J. Stettner, O. M. Magnussen, Structural Reorganisation of Pt(111) Electrodes by Electrochemical Oxidation and Reduction, J. Am. Chem. Soc. (111) (2017) jacs.7b01039. doi:10.1021/jacs.7b01039. 


\section{Annotated References}

$\left[12{ }^{* *}\right.$ : In situ surface $\mathrm{X}$-ray scattering studies of the initial stages of oxidation in perchloric and sulfuric acid solution. A complex potential-dependent sequence of changes in the adlayer structure and Pt surface relaxation was found. [11]*: In situ surface X-ray scattering measurements in perchloric acid solution, which indicate Pt surface buckling in the potential range positive of the butterfly peak.

[14 ${ }^{*}$ : Studies of the place exchange kinetics by in situ surface X-ray scattering in perchloric acid electrolyte. The data show that place exchange is fully reversible below a critical coverage and exhibits a logarithmic time dependence.

[48 ${ }^{* *}$ : First quantitative in situ grazing incidence small angle X-ray scattering studies of the Pt surface roughening induced by oxidation/reduction cycles in perchloric acid solution. The observed dependence of the characteristic lateral dimensions of the formed nanoscale islands on potential and cycle number are rationalized by a microscopic model based on the known surface dynamics under vacuum conditions.

[18 *: In situ surface-enhanced Raman studies of Pt oxidation. The interaction of perchlorate anions with adsorbed $\mathrm{OH}$ and the formation of peroxo- and superoxo-like surface oxides is reported.

${ }_{425}[43]^{* *}$ : Based on density functional theory calculations, a novel mechanism for the place exchange of a $\mathrm{Pt}$ surface atom with oxygen is suggested. It involves interactions with neighboring chemisorbed oxygen as well as with surface water. [46]*: Studies of Pt oxidation in perchloric acid solution by a combination of in situ X-ray scattering techniques. Detailed structural data on the oxide film at different potentials up to $1.6 \mathrm{~V}$ are reported. Step edge oxidation on cycled surfaces occurs at potentials as low as $0.8 \mathrm{~V}$. 


\section{Figure captions}

Figure 1: Typical cyclic voltammograms of $\mathrm{Pt}(111)$ in $0.1 \mathrm{M} \mathrm{HClO}_{4}$. The atomic-scale surface structures in the different potential regions are schemat-

435

Figure 2: In situ X-ray surface diffraction studies of $\mathrm{Pt}(111)$ oxidation in $0.1 \mathrm{M}$ $\mathrm{HClO}_{4}$. (a) Characteristic crystal truncation rods of the surface before (blue) and after (red) oxidation at $1.17 \mathrm{~V}$, indicating strong surface restructuring due

to place exchange. (b) CV and parallel X-ray intensity transient, showing place exchange at potentials $>1.05 \mathrm{~V}$ and full subsequent recovery of the surface structure after oxide reduction. (c) Kinetics of place exchange derived from SXRD studies, revealing an increase in the coverage of place exchange atoms by an $\ln (\mathrm{t})$ law (after Ref. 14]).

445

Figure 3: Results of in situ GISAXS studies on Pt(111) surface restructuring by oxidation/reduction cycles in $0.1 \mathrm{M} \mathrm{HClO}_{4}$. (a) Increase of characteristic distance between neighboring nanoscale Pt islands as a function of upper potential limit and number of cycles. In the upper panel, background-subtracted 450 GISAXS data are shown, which illustrate the intensity increase of the diffuse side wings after 5,10 , and 15 cycles to $1.62 \mathrm{~V}$, respectively, indicating vertical island growth (after Ref. [48]). (b) In operando GISAXS measurements of the time-dependent intensity profiles (top) and the integrated intensity of the diffuse side wings (bottom) during the 20th cycle to $1.37 \mathrm{~V}$. In the sweep in positive direction, two stages of the oxidation process are visible, i.e., a shallow decrease above $0.8 \mathrm{~V}$, which is attributed to step edge oxidation, followed by a steep intensity drop at $>1.05 \mathrm{~V}$ due to place exchange on $\mathrm{Pt}(111)$ terraces (after Ref. [46]). 


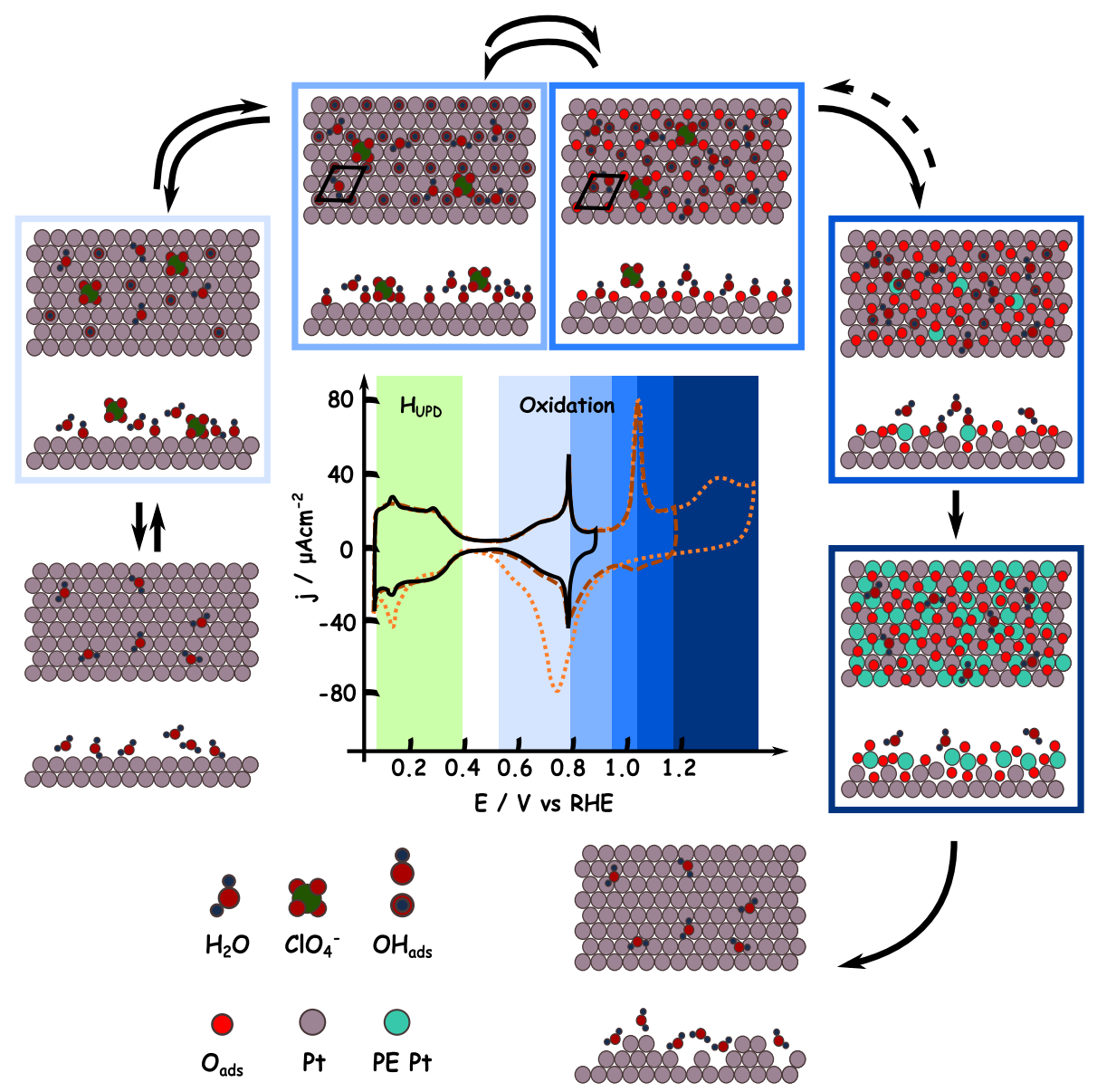

Figure 1: 

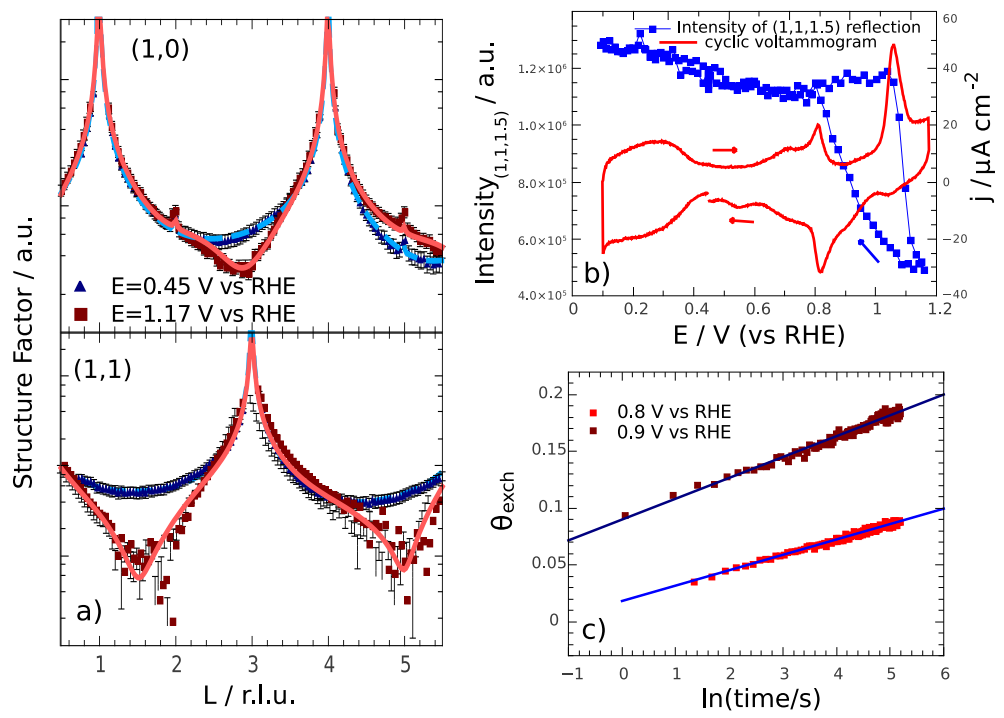

Figure 2:
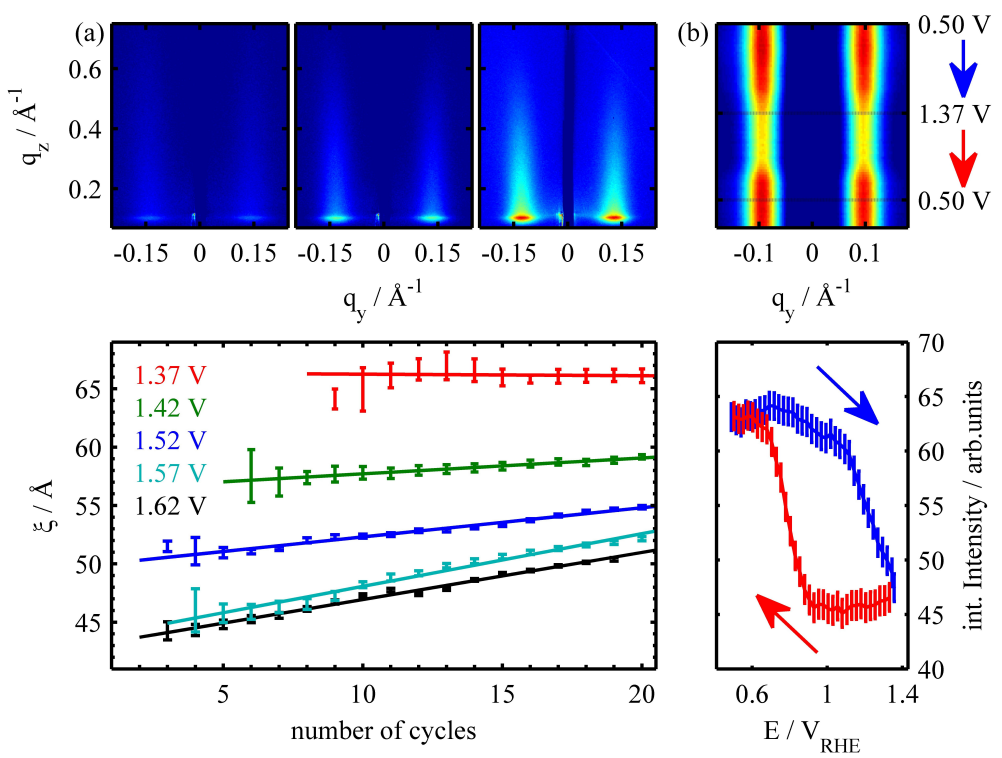

Figure 3: 